Ferrata Storti Foundation

\title{
DUX4r, ZNF384r and PAX5-P80R mutated B-cell precursor acute lymphoblastic leukemia frequently undergo monocytic switch
}

Haematologica 2021

Volume 106(8):2066-2075

\section{Correspondence:}

ESTER MEJSTRIKOVA

ester.mejstrikova@lfmotol.cuni.cz

Received: February 18, 2020.

Accepted: June 25, 2020.

Pre-published: July 9, 2020.

https://doi.org/10.3324/haematol.2020.250423

(C)2021 Ferrata Storti Foundation

Material published in Haematologica is covered by copyright. All rights are reserved to the Ferrata Storti Foundation. Use of published material is allowed under the following terms and conditions:

https://creativecommons.org/licenses/by-nc/4.0/legalcode. Copies of published material are allowed for personal or internal use. Sharing published material for non-commercial purposes is subject to the following conditions:

https://creativecommons.org/licenses/by-nc/4.0/legalcode, sect. 3. Reproducing and sharing published material for commercial purposes is not allowed without permission in writing from the publisher.

\author{
Michaela Novakova,,$^{1,2,3^{*}}$ Marketa Zaliova, ${ }^{1,2,3^{*}}$ Karel Fiser, ${ }^{1,2^{*}}$ Barbora \\ Vakrmanova, ${ }^{1,2}$ Lucie Slamova, ${ }^{1,2,3}$ Alena Musilova, ${ }^{1,2}$ Monika Brüggemann, ${ }^{4}$ \\ Matthias Ritgen, ${ }^{4}$ Eva Fronkova, ${ }^{1,2,3}$ Tomas Kalina, ${ }^{1,2,3}$ Jan Stary, ${ }^{2,3}$ Lucie \\ Winkowska, ${ }^{1,2}$ Peter Svec, ${ }^{5}$ Alexandra Kolenova, ${ }^{5}$ Jan Stuchly, ${ }^{1,2}$ Jan Zuna, ${ }^{1,2,3}$ \\ Jan Trka,,$^{1,2,3}$ Ondrej Hrusak ${ }^{1,2,3 \#}$ and Ester Mejstrikova ${ }^{1,2,3 \#}$
}

${ }^{1}$ CLIP - Childhood Leukemia Investigation Praguerague, Czech Republic; ${ }^{2}$ Department of Paediatric Hematology and Oncology, Second Faculty of Medicine, Charles University, Prague, Czech Republic; 'University Hospital Motol, Prague, Czech Republic; ${ }^{4}$ Department of Internal Medicine II, University Hospital Schleswig-Holstein, Kiel, Germany and ${ }^{5}$ Comenius University, National Institute of Children's Diseases, Bratislava, Slovakia

* $M N, M Z$ and KF contributed equally as co-first authors.

${ }^{*} \mathrm{OH}$ and EM contributed equally as co-senior authors.

\section{ABSTRACT}

$\mathrm{R}$ ecently, we described B-cell precursor acute lymphoblastic leukemia (BCP-ALL) subtype with an early switch to the monocytic lineage and the loss of the B-cell immunophenotype, including CD19 expression. Thus far, the genetic background has remained unknown. Among 726 children consecutively diagnosed with BCP-ALL, $8 \%$ patients experienced a switch detectable by flow cytometry (FC). Using exome and RNA sequencing, the switch was found to positively correlate with three different genetic subtypes: PAX5-P80R mutation (five cases with switch of five), rearranged (DUX4r) (30 cases of 41) and rearranged (ZNF384r) (four cases of ten). Expression profiles or phenotypic patterns correlated with genotypes, but within each genotype no cases who subsequently switched could be indentified. If switching was not taken into account, the B-cell-oriented FC assessment underestimated the minimal residual disease level. For patients with PAX5-P80R, a discordance between FC-determined and polymerase chain reactiondetermined minimal residual disease was found on day 15 , resulting from a rapid loss of the B-cell phenotype. Discordance on day 33 was observed in all the DUX4r, PAX5-P80R and ZNF384r subtypes. Importantly, despite the substantial phenotypic changes, possibly even challenging the appropriateness of BCP-ALL therapy, the monocytic switch was not associated with a higher incidence of relapse and poorer prognosis in patients undergoing standard ALL treatment.

\section{Introduction}

We recently described a pediatric B-cell precursor acute lymphoblastic leukemia (BCP-ALL) subtype with an early switch towards the monocytic lineage. ${ }^{1}$ Such a monocytic switch leads to a variable degree of monocytosis in the early phase of treatment. The switched monocytic cells ("monocytoids") have significantly diminished expression of immature or lymphoid markers (i.e., CD19 and CD34) and upregulated myeloid markers (i.e., CD33 and CD14) while keeping shared immunoglobulin/T-cell receptor (IG/TR) rearrangements with malignant B lymphoblasts. The increase in monocytoid cells was most prominent at days 8 and 15 after the start of therapy, although the proportion differed between the patients. We reported frequent expression of CD2 and a higher prevalence of ERG deletions and IKZF1 gene alterations in this leukemia subtype than in other subtypes. ${ }^{1}$ However, at that time, we 
were unable to uncover the genetic background. In our previous work, we observed a slower response to initial treatment in patients with a monocytic switch than in patients without it. Despite significant changes in the phenotype towards the monocytic lineage in some of the patients, riskbased ALL treatment remained the treatment of choice. ${ }^{1}$

Recently, new genetic subtypes of ALL, particularly within the "B-other" group, were discovered using RNA sequencing (RNA-seq)., ${ }^{2,3}$ We investigated whether any of the newly defined subsets had a higher tendency to undergo monocytic switch.

Monocytic switch is accompanied by the gradual loss of CD19. As the determination of minimal residual disease (MRD) by flow cytometry (FC) relies on B-cell markers, switching leads to MRD underestimation. The FC MRD value on day 15 is used for patient stratification in the current pediatric Berlin-Frankfurt-Münster (BFM) BCP-ALL treatment protocols. It is an open question whether FC monitoring of MRD should be adapted for patients with switch.

Recently, anti-CD19 therapy, namely, blinatumomab, was added to pediatric ALL frontline treatment protocols, and a larger proportion of patients will be treated with it in the near future. As patients with switch that includes CD19 loss may be selected for anti-CD19 treatment, knowledge about subtypes prone to switch will be of interest. Moreover, anti-CD19 therapy may result in monocytic switch, as was repeatedly reported, especially for cases of KMT2A rearrangement. ${ }^{4-9}$

The aim of this study is to describe the molecular landscape of ALL with monocytic switch in the context of newly discovered genetic subtypes. We aim to describe the switching behavior in distinct genetic subtypes and to analyze the extent of its influence on the FC assessment of MRD. In addition, we analyzed the impact of switching phenomenon on relapse risk.

\section{Methods}

This study was approved by the Institutional Review board of the University Hospital Motol and the Second Faculty of Medicine and informed consent was obtained from all patients and their guardians in accordance with the Declaration of Helsinki.

We included 726 BCP-ALL patients (age, 0-18 years) diagnosed in the Czech Republic between 09/2007 and 02/2019. Patients were treated according to the following BFM protocols: ALL-ICBFM-2002 ( $\mathrm{n}=17)$; ALL BFM 2000 ( $\mathrm{n}=177)$; ALL BFM 2009 ( $n=483$ ); the Interfant 2006 protocol (for children younger than 12 months; $\mathrm{n}=30$ ); the EsPhALL protocol (for BCR-ABL1-positive patients; $n=16)$; and other protocols ( $n=3$; one patient was treated by a modified protocol, one Down syndrome patient with significant comorbidities was treated with a reduced ALL BFM 2009 protocol, and one patient moved abroad after induction therapy). Some of the 726 patients (179 of 726 ) diagnosed between 09/2007 and $05 / 2010$ were included in a previous study. ${ }^{1}$ For selected analyses, we expanded this consecutive cohort with additional 19 patients who were diagnosed before 09/2007 and for whom the switching phenomenon was identified and described retrospectively $(\mathrm{n}=16)^{1}$ or who were diagnosed abroad (Germany, $\mathrm{n}=2$; and Slovakia, $\mathrm{n}=1$ ) (Online Supplementary Figure S1). All patients were screened for the presence of recurrent fusion genes (BCR-ABL1, KMT2A-AFF1, ETV6-RUNX1 and TCF3-PBX1). In all patients, standard cytogenetic evaluation and assessment of the DNA index were performed as published previously.,10 The B-other subset was defined as BCP-ALL by the absence of all routinely investigated classifying aberrations (ETV6-RUNX1, hyperdiploidy, hypodiploidy, BCR-ABL1, KMT2A rearrangements and TCF3PBX1).

\section{Flow cytometry}

The diagnostic phenotype was determined through standard protocols. ${ }^{11-13}$ Ambiguous lineage acute leukemia (ALAL) diagnosis was based on the European Group for the Immunological Characterization of Leukemias (EGIL) criteria ${ }^{11,12,14,15}$ and/or the World Health Organization ${ }^{16}$ classification. A summary of the antibody clones and vendors is presented in the Online Supplementary Table S1. In addition, the percentage of the B-monocytoid population defined as $\mathrm{CD} 19^{\text {pos }} \mathrm{CD} 14^{\text {pos }}$ was determined with an eight-color combination of antibodies against CD45, CD14, CD34, CD19, CD33, CD20, CD10 and CD3 at diagnosis (day zero $[\mathrm{d} 0]$ ), day $8[\mathrm{~d}+8]$, day $15[\mathrm{~d}+15]$, and day $33[\mathrm{~d}+33]$ in the bone marrow (BM) and/or peripheral blood ( $\mathrm{PB})$ as shown previously. ${ }^{1} \mathrm{FC}$-assessed $\mathrm{MRD}$ was evaluated using three- or fourcolor monoclonal antibody combinations in the period between 1998 and $2007^{17}$ and using eight-color combinations starting in 2007. 18,19 The sensitivity (level of quantification and level of detection, LoO and LoD, respectively) of the FC-assessed MRD was defined by the number of nucleated cells measured in an MRDspecific tube (for a sensitivity of $10^{-3}$ and $10^{-4}, 20,000$ and 200,000 nucleated cells were measured, respectively). Only samples with an appropriate FC MRD sensitivity were included in the analysis: generally, a sensitivity of $10^{-4}$ was required; for cases with polymerase chain reaction (PCR)-determined $M R D \geq 10^{-2}$, measures of sensitivity that were at least one log value lower than that of the actual PCR-determined MRD value were sufficient. The sample was assessed as FC MRD positive when a cluster of at least 20 events with an aberrant B-cell phenotype was detected.

\section{Definition of the switching phenomenon}

Based on our previous work, ${ }^{1}$ we defined the switching phenomenon as the presence of an intermediate B-monocytoid population, i.e., BCP-ALL blasts with a gradual decrease in CD19 expression accompanied by a gradual increase in the expression of at least one monocytic marker (CD14, CD33, or higher side scatter, SSC) (Online Supplementary Figure S2) at any time point between $\mathrm{d} 0$ and $\mathrm{d}+33$. We used fluorescence-activated cell sorting (FACS) of the intermediate B-monocytoid (CD19 $\left.9^{\text {pos }} \mathrm{CD} 14^{\mathrm{pos}}\right)$ and monocytoid (CD19 $\left.{ }^{\text {nes }} \mathrm{CD} 14^{\mathrm{pos}}\right)$ populations to show that the IG/TR rearrangements were identical to those in $\mathrm{B}$ lymphoblasts when enough material was available (patients $n=37$; samples $n=70$ ) between $\mathrm{d} 0$ and $\mathrm{d}+33$.

\section{Immunoglobulin/T-cell receptor polymerase chain reaction-assessed minimal residual disease}

Patient-specific IG/TR assays were developed as described previously. ${ }^{1,20,21}$ In patients with two independent IG/TR targets, the higher value was reported. Sensitivity (LoD) and quantitative range (LoQ) for each assay was defined according to the European Study Group on MRD guidelines. ${ }^{22}$ A minimum LoD of $10^{-4}$ was achieved in all patients/timepoints.

\section{Statistics}

Fisher's exact test was used for comparing categorical variables, and the Mann-Whitney test was used for continuous variables. Other tests used are explicitly indicated in the text. The results were considered significant when $P$-values were less than 0.05 . Statistical analyses were performed using GraphPad software (GraphPad Software, Inc., La Jolla, CA, USA), $\mathrm{R}^{23}$ and StatView version 5.0 (StatView Software, Cary, NC, USA). 
Data analysis of genetic and immunophenotypic data

RNA-seq data (patients with switch, $\mathrm{n}=73$; patients without switch, $n=124$ ), whole-exome sequencing (WES) data (patients with switch, $\mathrm{n}=30$; patients without switch, $\mathrm{n}=70$ ), singlenucleotide polymorphism (SNP) data (patients with switch, $\mathrm{n}=59$; patients without switch, $n=108$ ) and ERG deletion data were analyzed in diagnostic samples as published previously. ${ }^{24-26}$ Diagnostic samples were sorted if blasts comprised fewer than $80 \%$ of mononuclear cells. Purity of sorted populations was at least $90 \%$. Patients in the B-other group without the following aberrations were assigned to the B-other rest subgroup: DUX4, ZNF384, MEF2D and NUTM1 rearrangements; BCR-ABL1-like and ETV6RUNX1-like expression profiles; and IAMP21, PAX5-P80R and IKZF1-N159Y mutations.

In order to analyze RNA-seq and immunophenotypic data, uniform manifold approximation and projection (UMAP) ${ }^{27}$ was used as the dimensionality reduction algorithm. Hierarchical clustering analysis (HCA) was performed using Euclidean distance and Ward's linkage.

\section{Results}

\section{Incidence and features of patients with monocytic switch}

Prospectively, we identified 61 patients with monocytic switch using the criteria described above (Table 1), which corresponded to $8 \%$ of patients.

No sex difference occurred in the monocytic switch $(69 \%$ females vs. $56 \%$ males, not significant [n.s.]) but the monocytic switch was associated with older age at diagnosis (median 7.8 vs. 4.5 years, respectively, $P<0.001$ ) and a higher initial white blood cell (WBC) count (median 10,750/uL

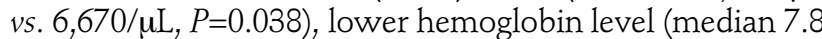
$\mathrm{g} / \mathrm{dL}$ vs. $8.8 \mathrm{~g} / \mathrm{dL}, P=0.0046$ ), higher platelet count (median $74,000 / \mu \mathrm{L}$ vs. $58,000 / \mu \mathrm{L}, P=0.048)$ and a higher proportion of blasts in $\mathrm{PB}(59 \%$ vs. $32 \%, P=0.0039)$, while the proportion in BM did not differ $(91.2 \%$ vs. $90 \%$, n.s.).

We confirmed the presence of patient-specific IG/TR rearrangements in the sorted monocytoid cells in 33 of 37 patients in whom the sorting was successful at various time points between $\mathrm{d} 0$ and $\mathrm{d}+33$ ( 16 of 19 positive at $\mathrm{d} 0 ; 23$ of 27 positive between $d+1$ and $d+14$; 14 of 15 positive at $d+15$; eight of nine positive between $d+16$ and $d+33)$. In the morphological examination of some patients, an increase in monocytic cells with variable morphology (monoblasts, promonocytes, and mature monocytes) was very clear. In three patients, at $d+8$ (DUX4r, n=2; and ZNF384r, $n=1$ ), we observed over 10,000 monocytes/ $\mu \mathrm{L}$ in the PB samples (Online Supplementary Figure S3A and B).

\section{Monocytic switch is most frequent in the PAX5-P80R, DUX4r, and ZNF384r genetic subtypes}

In order to study the relationship between monocytic switch and genetic background, routine (cyto)genetic investigations were supplemented with a retrospective analysis using RNA-seq data, enabling a more detailed genomic characterization of the ALL patients. Patients with monocytic switch were unequally distributed across the ALL subtypes in the unselected consecutive cohort (chi square test $P<0.0001$; Table 1); they were significantly enriched in the PAX5-P80R-, DUX4r- and ZNF384r--positive ALL subtypes

Table 1. Distribution of cases with monocytic switch in an unselected cohort of B-cell precursor acute lymphoblastic leukemia patients ( $\mathrm{n}=726$ ) stratified into genetic/biological subtypes.

\begin{tabular}{|c|c|c|c|c|}
\hline \multirow{2}{*}{\multicolumn{2}{|c|}{ BCP-ALL subtypes }} & \multicolumn{2}{|c|}{ Monocytic switch } & \multirow[t]{2}{*}{$\mathbf{P}$} \\
\hline & & $\begin{array}{l}\text { No } \\
\text { n (\%) }\end{array}$ & $\begin{array}{c}\text { Yes } \\
\text { n (\%) }\end{array}$ & \\
\hline \multicolumn{2}{|c|}{$\mathrm{HHD}^{1}$} & $266(97)$ & $8(3)$ & $P<0.0001$ \\
\hline \multicolumn{2}{|c|}{ ETV6-RUNX1 } & $180(99)$ & $1(1)$ & $P<0.0001$ \\
\hline \multicolumn{2}{|c|}{ KMT2Ar } & $29(88)$ & $4(12)$ & ns \\
\hline \multicolumn{2}{|c|}{ TCF3-PBX1 } & $27(100)$ & $0(0)$ & ns \\
\hline \multicolumn{2}{|c|}{$B C R-A B L 1$} & $13(76)$ & $4(24)$ & ns \\
\hline \multicolumn{2}{|c|}{ Hypodiploidy ${ }^{2}$} & $8(89)$ & $1(11)$ & ns \\
\hline \multirow{11}{*}{ 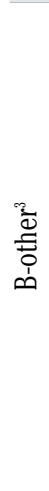 } & B-other rest ${ }^{4}$ & $49(94)$ & $3(6)$ & ns \\
\hline & DUX4r & $11(27)$ & $30(73)$ & $P<0.0001$ \\
\hline & BCR-ABL1-like & $18(95)$ & $1(5)$ & ns \\
\hline & ZNF384r & $6(60)$ & $4(40)$ & $P=0.0022$ \\
\hline & ETV6-RUNXI-like & $10(100)$ & $0(0)$ & ns \\
\hline & iAMP21 & $6(100)$ & $0(0)$ & ns \\
\hline & PAX5-P80R & $0(0)$ & $5(100)$ & $P<0.0001$ \\
\hline & NUTM1r & $4(100)$ & $0(0)$ & ns \\
\hline & $M E F 2 D r$ & $3(100)$ & $0(0)$ & ns \\
\hline & IKZF1-N159Y & $1(100)$ & $0(0)$ & ns \\
\hline & Unknown $^{5}$ & $34(100)$ & $0(0)$ & ns \\
\hline \multicolumn{2}{|c|}{ Total } & $665(92)$ & $61(8)$ & \\
\hline
\end{tabular}


but were significantly less frequent among high-hyperdiploid subtypes and extremely rare in ETV6-RUNX1-positive ALL. In addition to 61 patients with monocytic switch in the consecutive cohort, we identified another 19 patients with monocytic switch who belonged to the DUX4r $(\mathrm{n}=15)$, PAX5-P80R ( $=2), Z N F 384 \mathrm{r}(\mathrm{n}=1)$ and high-hyperdiploid ( $n=1)$ ALL subtypes. Nevertheless, except for the DUX4r ALL subtype (representing the subgroup with the highest prevalence of switch, with nearly half of all patients having monocytic switch [30 of 61]), the number of patients was too low (and/or the genomic data were too limited) to study the impact of the broader genomic context on monocytic switch occurrence within the individual subtypes. In the DUX4r ALL subtype group, we did not find any association between monocytic switch and the most frequent secondary genetic aberrations (deletions of $E R G$, $C D K N 2 A / B, I K Z F 1$ or PAX5 or mutations in the NRAS or KMT2D genes; Online Supplementary Table S2).

The pattern of monocytic switch correlated with the genotype (Figure 1). Patients with the DUX4r ALL subtype presented with predominant CD19 positive (CD19 $9^{\text {pos }}$ Bprecursor blasts at diagnosis and typically maintained these cells, while the phenotype gradually became more monocytic on $d+8$ and $d+15$. Among the 25 patients with switching phenomenon identified already at diagnosis, only two patients were of the DUX4r subtype (Fisher's exact test $P=0.0002)$. Patients with the PAX5-P80R mutation also presented with CD19 ${ }^{\text {pos }} B$ precursor blasts, but after $d+8$, the Bcell markers had typically disappeared concomitantly during the switch. Patients with the ZNF384 fusion often coexpressed B-precursor and monocytic markers at diagnosis or presented with bilineal disease with separate monocytoid population of blasts, and the monocytoid population often became more prominent during chemotherapy.

The amplitude of monocytic switch (determined as the maximum number of intermediate B-monocytoid cells) did not differ among the different genetic subtypes (Online Supplementary Figure S3C, Kruskal-Wallis test, n.s.).

\section{Differences in the diagnostic gene expression signature associated with a subsequent monocytic switch are driven by genotype}

We used RNA-seq to assess changes in gene expression that were related to monocytic switch at the time of diagnosis. We analyzed 197 diagnostic transcriptomes and determined the genes that were differentially expressed among patients with $(\mathrm{n}=73)$ and without monocytic switch $(n=124)$. Among the 50 top-ranking genes (only proteinencoding genes with absolute fold changes $\geq 2.5$ were considered; Online Supplementary Table S3), we found the pro-
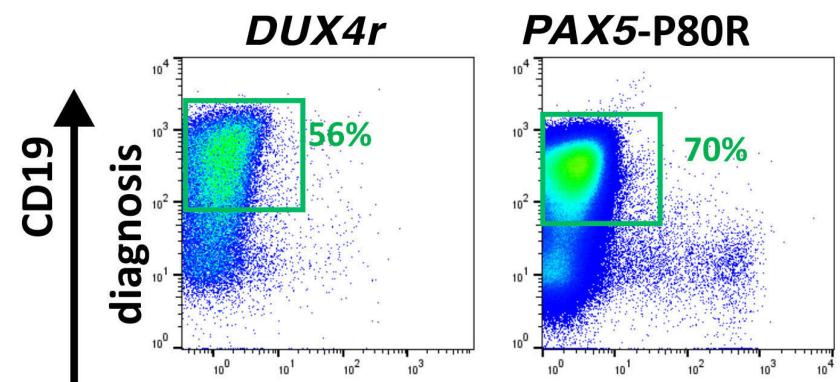

ZNF384r

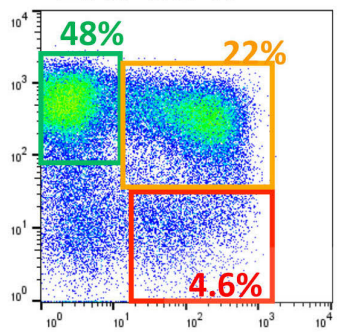

ZNF384r bilineal
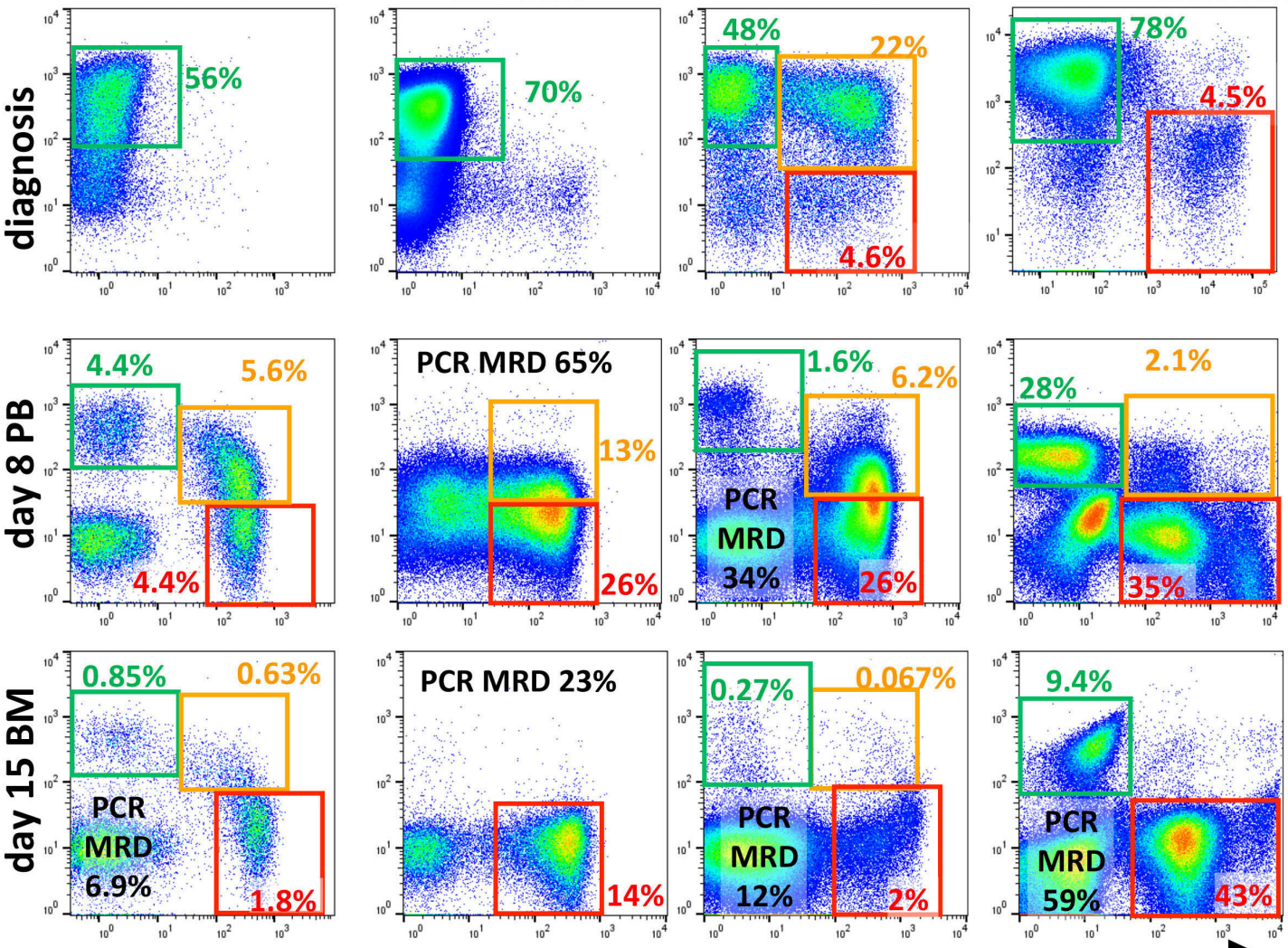
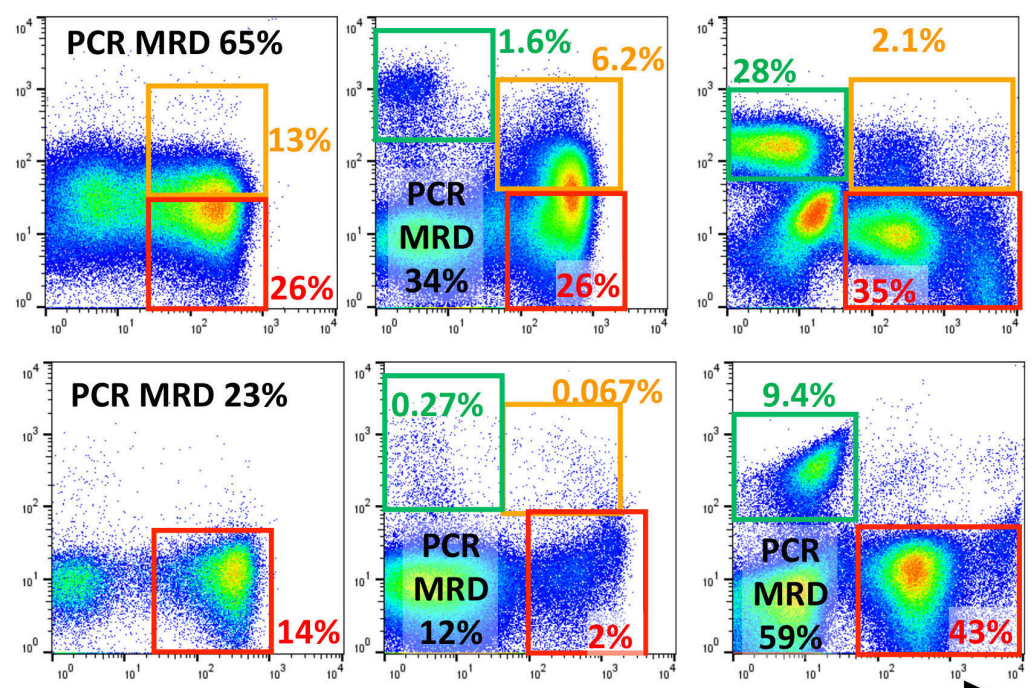

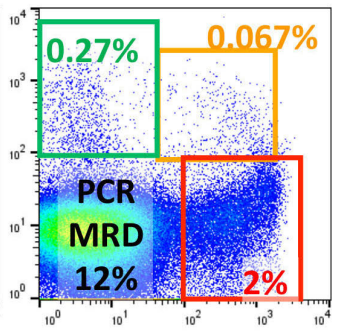

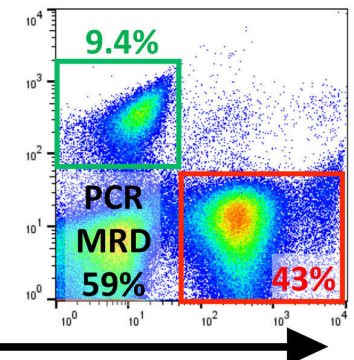

CD14

Figure 1. Monocytic switch appearance. In cytometric plots, mononuclear cells after exclusion of doublets and nonmalignant T cells and B cells (if available) are shown. Green, orange and red rectangles highlight the preswitched B-precursor blasts, B-monocytoid intermediate cells and fully switched monocytoids, respectively. Examples of patients with the respective genotypes are shown at day zero (d0) (bone marrow [BM]), $d+8$ (peripheral blood [PB]) and $d+15$ (BM). The percentage of each population is shown (of all nuclear cells) in the corresponding color. Polymerase chain reaction (PCR)-determined minimal residual disease (MRD) values in percentages are shown in black. We observed DUX4r monocytic switch pattern in 43 of 45 patients, PAX5-P8OR pattern in seven of seven patients and ZNF384r pattern in three of five patients within the respective genotypes in all patients analyzed $(n=745)$. 
liferation-activating gene CRLF2 (lower in patients with monocytic switch than in those without monocytic switch), the cell cycle regulator CCNA1 (cyclin A1, higher in patients with monocytic switch) and androgen receptor (AR, higher in patients with monocytic switch), as well as six genes with a CD marker designation: CD371, CD301, CD1E, CD125 (all higher in patients with monocytic switch), CD158K and CD20 (both lower in patients with monocytic switch). Consistent with our previously published data, patients with switch had increased CEBPA expression $(P<0.0001)$, which was also the case when only DUX4r patients were analyzed $(P<0.0001)$.

We asked whether these changes were primarily driven by monocytic switch itself or by the underlying genotype. Both the unsupervised hierarchical clustering and the UMAP analyses showed that the patients primarily clustered according to genotype, including the DUX4r, PAX5P80R and ZNF384r subtypes, which were enriched for monocytic switch, and whether the patients eventually experienced monocytic switch seemed to be secondary (Figure 2A; Online Supplementary Figure S4).

As genotype-associated transcriptome differences may override monocytic switch-associated differences, we repeated the analysis in the most prevalent (accounting for $>60 \%$ of the patients with switch) genotypic subset (DUX4r). The Online Supplementary Figure S5 shows that the clustering of the switching patients was not clear. Among the top-ranking most differentially expressed genes between switching $(n=44)$ and nonswitching $(n=11)$ patients were the previously described $C E B P A,{ }^{1}$ hematopoietic regulator FLT3 and Toll-like receptor TLR10, which were all higher in patients with the DUX4r subtype with switch than in patients with DUX4r without switch (Online Supplementary Table S4).

\section{Genotype also influences immunophenotype associated with subsequent monocytic switch}

We next sought to determine whether the diagnostic immunophenotype predicts, similarly to transcriptome, monocytic switch behavior and whether it can be associated with underlying genetic aberrations.

For these purposes we analyzed the diagnostic immunophenotype of the blast population in 745 patients.

The overall picture appeared to be analogous to that of the transcriptome data: diagnostic immunophenotypes were grouped based on the genetic subtype as seen in the UMAP analysis rather than by the monocytic switch status (Figure 2B).

In our previous study all patients with monocytic switch harbored CD2 expression, although belonging to different genetic subtypes. As we have shown recently, ${ }^{28} \mathrm{CD} 2$ is expressed in approximately $75 \%$ of DUX $4 \mathrm{r}$ patients whereas a newly described marker CD371 (CLL-1) is found in all patients with DUX4r (Online Supplementary Figure S8).

We also observed the expression of CD2 and CD371 in five of seven and two of five patients with PAX5-P80R, respectively. Interestingly, all seven patients with $P A X 5$ P80R had homogeneous expression of CD66c. Expression of CD4, a rare aberrant marker in BCP-ALL, was present in four of seven $P A X 5$-P80R patients, all of which had cells with a phenotype of $\mathrm{CD} 34^{\text {neg }} 33^{\text {pos }}$ (in contrast to the other three of seven $\mathrm{CD} 4{ }^{\text {neg }} \mathrm{CD} 34^{\text {pos }}$ patients). Interestingly, CD66c expression was retained on switched monocytoid cells on $\mathrm{d}+15$ in all six patients with available data.

Patients with the ZNF384r subtype were often classified as having acute leukemia of ambiguous lineage (ALAL) using the EGIL classification (six of 11 patients).

The typical immunophenotypes of the main three subtypes with switch (DUX4r: $\mathrm{CD} 10^{\text {pos }} \mathrm{CD} 20^{\text {nes }} \mathrm{CD} 34^{\text {pos }} \mathrm{CD} 22^{\text {pos }} \mathrm{CD} 371^{\text {pos }}$; $\quad$ PAX5-P80R: $\mathrm{CD} 10^{\text {neg } / \text { pos }} \mathrm{CD} 66 \mathrm{c}^{>75 \%} \mathrm{CD} 2^{<50 \%} \mathrm{CD} 4^{\text {neg }} \mathrm{CD} 34^{\text {pos }} \mathrm{CD} 33^{<50 \%}$ or $\mathrm{CD} 1{ }^{\text {neg }}{ }^{\text {pos }} \mathrm{CD} 66 \mathrm{c}^{>75 \%} \mathrm{CD} 2^{\text {pos }} \mathrm{CD} 4{ }^{\text {pos }} \mathrm{CD} 34^{\text {neg }} \mathrm{CD} 33^{\text {pos; }}$ ZNF384r: $\left.\mathrm{CD} 10^{<50 \%} \mathrm{CD} 13^{\text {pos }} \mathrm{CD} 66 \mathrm{c}^{\text {neg }} \mathrm{CD} 34^{\text {pos }} \mathrm{CD} 135^{\text {pos }} \mathrm{CD} 24^{<60 \%}\right)$, are shown in the Online Supplementary Figure S8.

\section{Discordance between flow cytometry- and polymerase chain reaction-determined minimal residual disease reflects different switching dynamics in individual genetic subtypes}

Loss of B-cell markers during monocytic switch interferes with B-cell-oriented MRD analysis by FC. As we showed previously in our pilot study ${ }^{1}$ and now in a significant cohort of patients, switched monocytoid blasts maintain leukemic IG/TR rearrangements despite completely losing the B-cell phenotype. We thus analyzed the influence of switch on MRD detection by comparing FC and PCR MRD quantitation results in the genotype subsets most prone to switch.

We had only limited data for $\mathrm{d}+8 \mathrm{~PB}$ samples from PAX5P80R ( $n=3)$ and ZNF384r patients $(n=2)$. Spearman $R$ for DUX $4 r(\mathrm{n}=31)$ and patients with monocytic switch outside these three genetic subtypes $(n=11)$ was 0.7 and 0.72 , respectively (Online Supplementary Figures S6 and S7).

But, as shown in Figure 3, FC underestimation of MRD led to pronounced discordance at $\mathrm{d}+15$ in the PAX5-P80R patients. The concordance of the FC and PCR MRD positivity/negativity at the $10^{-3}$ level was $91 \%$ for the DUX $4 r$ subtype and $82 \%$ for the ZNF384r subtype but only $17 \%$ for the PAX5-P80R subtype. Concordance at the $10^{-1}$ level, which was determined at this time point as the FC cut-off value for stratifying patients for undergoing high-risk treatment according to the BFM protocols, was $78 \%$ for the DUX4r subtype and 78\% for the ZNF384r subtype but only $43 \%$ for the PAX5-P80R subtype.

At $d+33$, all three subtype groups showed poor correlation between the FC and PCR MRD values (Figure 3 ) compared to previously published data. ${ }^{18,29}$ The concordance of the FC- and PCR-determined MRD at the level of $10^{-3}$ was $55 \%$ for the DUX4r subtype, 56\% for the ZNF384r subtype and $33 \%$ for the PAX5-P80R subtype.

Monocytic switch rarely caused MRD discrepancies in the other genetic subtypes. When analyzed separately, among 23 patients in the DUX4r reg $P A X 5-P 80 R^{\text {neg }} Z N F 384 r^{\text {neg }}$ subset and in which monocytic switch was observed, FC $M R D$ at the appropriate level of sensitivity (defined in the Methods) was measured in 21 and 16 patients on $\mathrm{d}+15$ and $\mathrm{d}+33$, respectively (Online Supplementary Figure S7). The concordance at $\mathrm{d}+15$ for the $10^{-1}$ and $10^{-3}$ levels was $86 \%$ and $90 \%$, respectively. The concordance at $d+33$ at the $10^{-3}$ level was $76 \%$.

At week plus 12 majority of samples were PCR (86\%) and FC (97\%) MRD negative. Concordance for DUX4r ( $\mathrm{n}=44)$, PAX5-P80R ( $\mathrm{n}=5), Z N F 384 \mathrm{r}(\mathrm{n}=8)$ and cases with switch outside these subtypes $(n=21)$ was $82 \%, 80 \%, 100 \%$ and $90 \%$, respectively (Online Supplementary Figures S6 and S7).

\section{Prognostic relevance of monocytic switch}

From a clinical perspective, it is important to know whether monocytic switch is of prognostic relevance and whether the phenotype changes during relapse. All but one 
patient with monocytic switch was treated according to standard BCP-ALL frontline protocols. In the whole study cohort, eight of 80 patients with monocytic switch relapsed. Of the eight relapsed patients, six patients relapsed with an identical BCP-ALL subtype to the subtype at diagnosis (two had the $B C R-A B L 1$ subtype, one had the DUX4r subtype, one had the HHD subtype, one had the
ZNF384r subtype, and one had the B-other rest subtype). Interestingly, only one of those patients (ZNF384r) developed monocytic switch during induction therapy for relapse. Two patients (one with the DUX4r subtype and one with the KMT2Ar subtype) relapsed with monocytic AML. In all three patients with the presence of monocytoid blasts at relapse, we proved IG/TR rearrangements in the
A

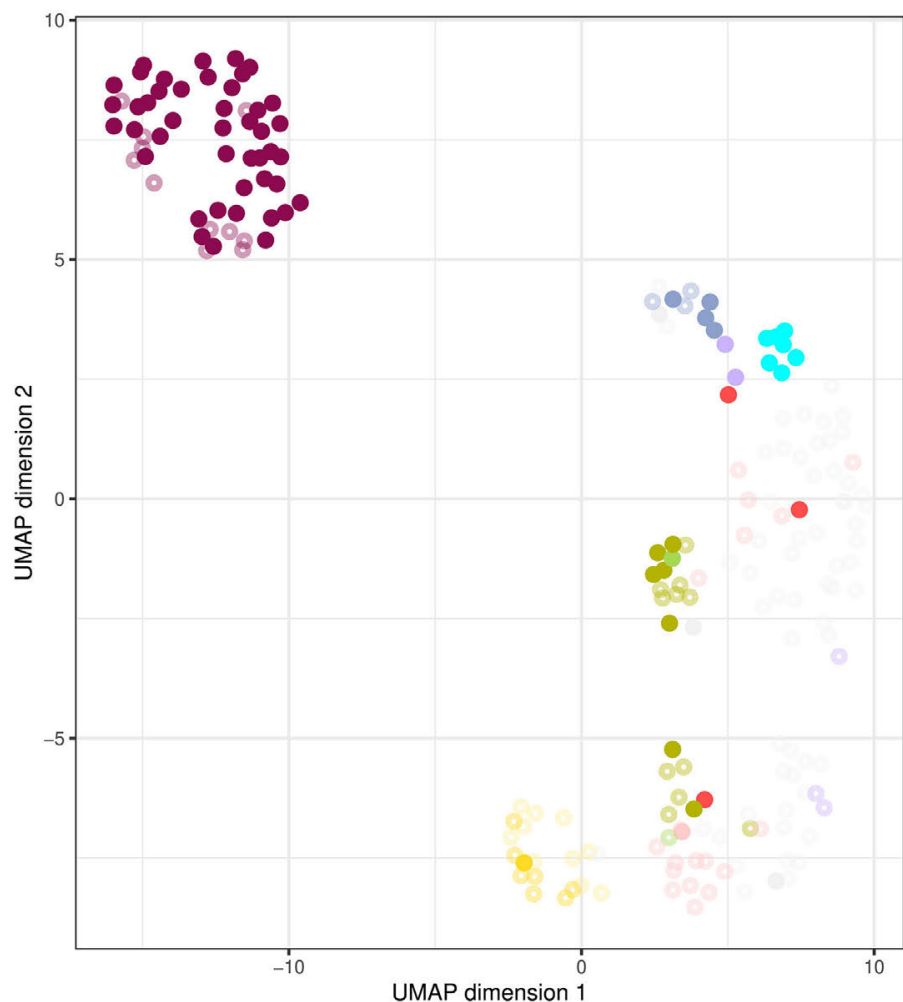

B

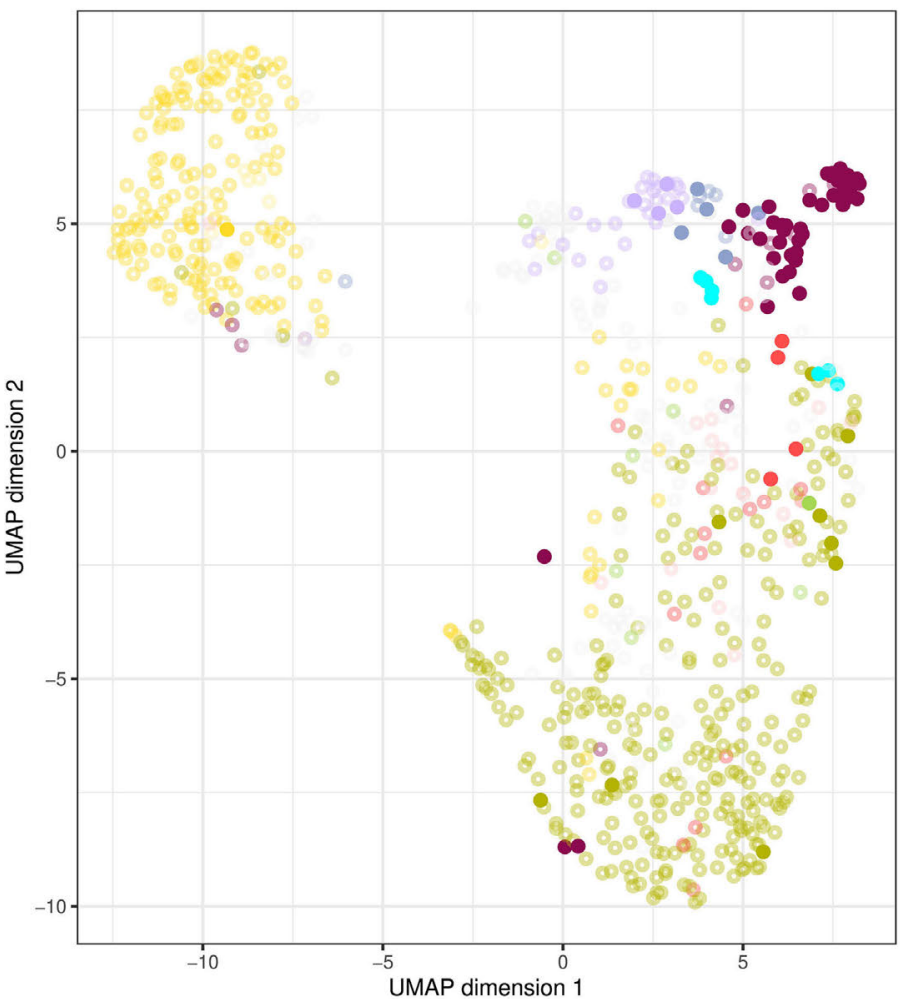

genetic subtype

- BCR-ABL1

- $B C R-A B L 1-$ like

- DUX4r

- ETV6-RUNX1

ETV6-RUNX1-like

- $H H D$

- $H Y P O$

- KMT2Ar

other subtypes

- PAX5-P80R

- ZNF384r

\section{switching cases}

- no

- yes

Figure 2. Multidimensional analysis of RNA sequencing and flow cytometry data. (A) Overview of the gene expression found for all patient ( $n=197$ ) using UMAP as the dimensionality reduction algorithm for RNA sequencing data. Only the genes with the most variability (genes with an SD 0.4-fold higher than the maximum SD, $n=271$ ) were used. Patients with switch are shown as full circles, and patients without switching are shown as empty circles. Relevant genetic subtypes are shown in color. (B) Overview of immunophenotypes of all cases $(n=745)$ using UMAP as dimensionality reduction algorithm. Blasts were gated using common backbone (forward scatter, side scatter, CD45). The data (expression of markers on blast population in percentage) were prepared from multiple tubes and merged together. Missing values were replaced with marker median values. Open circles represent cases without monocytic switch, full circles represent cases which developed switching. Relevant genetic subtypes are shown in color. 
monocytoid cells identical to those in the original BCP-ALL clone. There was no difference in the event-free survival (EFS) between the prospective patients with or without monocytic switch (5-year EFS, $82 \pm 5.5 \%$ and $86 \pm 1.6 \%$, respectively, Figure 4) also when only patients in the high risk/slow early response group were considered (Online Supplementary Figure S9).

\section{Discussion}

Monocytic switch provides evidence for the relationship between the monocytic and lymphoid lineages. Rigid models of hematopoiesis assume early separation of the monocytic and lymphoid lineages, but there is increasing evidence of innate immune functions in lymphoid lineages, including phagocytosis. ${ }^{30}$ The biological origin of the phenomenon remains to be clarified. Rarely B-cell malignancies of various stages of differentiation under unknown circumstances can undergo transdifferentiation into myeloid/histiocytic malignancies. ${ }^{31,32}$ An interesting area for future investigation is the relationship between of monocytic cells to their $\mathrm{CD} 14^{\text {pos }}$ dendritic cells counterparts. CD2, a frequent aberrant marker of BCP ALL blasts prior to monocytic switching, does not clarify their fate as it is known to be expressed in subsets of both dendritic cell $\mathrm{s}^{33}$ and monocytic AML. ${ }^{34}$ The constant role of the transcription factor C/EBP $\alpha$ seems to play a role in the process. In our previous work, we found higher expression of CEBPA in patients with monocytic switching, which was also true in this extended cohort. C/EBP $\alpha$ directly represses B-cell genes. DiStefano et al. reported that $\mathrm{C} / \mathrm{EBP} \alpha$ can enforce B-cell transcription factor silencing by increasing the expression of the histone demethylase Lsd1 (Kdm1a) and the histone deacetylase Hdac1 at the protein level and that these enzymes are required for the downregulation of $\mathrm{B}$-cell enhancers and the silencing of the B-cell program. ${ }^{35}$ In vitro models demonstrate that $\mathrm{C} / \mathrm{EBP} \alpha$ induces the repression of key B-cell regulators such as Foxo1, Ebf1 and Pax $5^{36}$.

In our study we show that monocytic switch behavior is not limited to a single genotypic subset and that various leukemia genotypes show different propensities to switch to monocytic cells. In agreement with our previous report, in this study, the majority of patients whose blasts switched to monocytoids were categorized in the B-other subset ( $70 \%$ of patients with monocytic switch compared to $21 \%$ of patients without switch; Fisher's exact test $P<0.00001$ ). New genomic methods - namely, RNA-seq - were recently used to discover new recurring genetic subtypes within the mixture of the genotypes thus far labeled B-other. ${ }^{2,3,24,2,5,37-40}$ Three of these subsets, DUX4r, PAX5-P80R, and ZNF384r, were frequently associated with switch, constituting the majority of the patients with switch in this study. We did not identify a known subtype-defining genetic aberration (B-other rest) in only $3.8 \%$ of the patients with monocytic switch (Table 1). Interestingly, all patients with PAX5-P80R presented with monocytic switch.

Although it did not reach statistical significance (probably due to the low number of patients), a switching phenomenon was observed in $24 \%$ of cases with BCR-ABL1. McClellan et al. found that primary $B C R-A B L 1$-positive BCP-ALL cells could be reprogrammed into macrophagelike cells through exposure to cytokines in vitro or by transient expression of the transcription factor $\mathrm{C} / \mathrm{EBP} \alpha$ or PU.1. ${ }^{41}$ Surprisingly, monocytic switch was not significantly more frequent in the KMT2Ar subtype than in other sub-

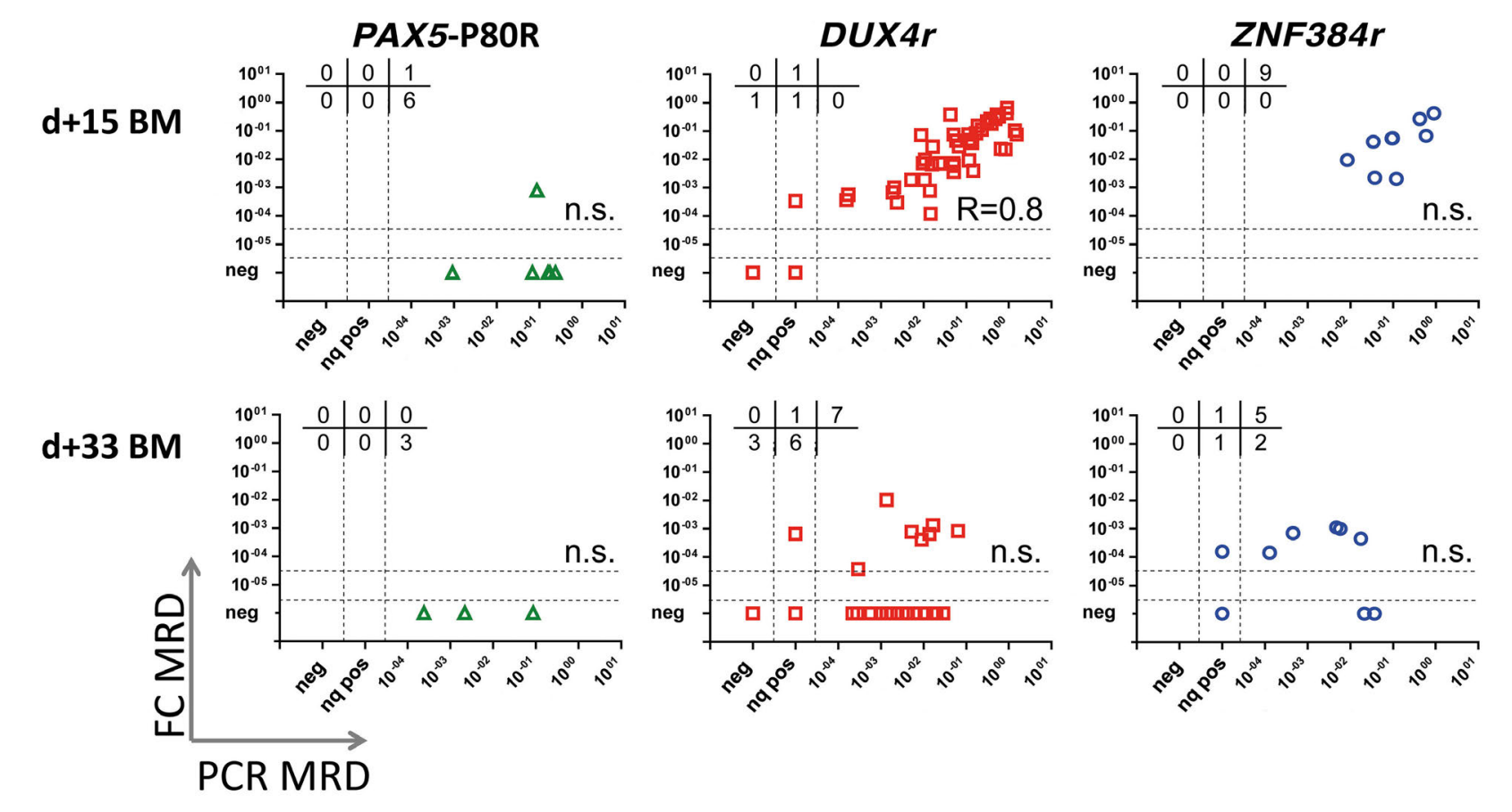

Figure 3. Correlation of the mimal reidual disease results obtained by flow cytometry and polymerase chain reaction in selected patient groups. Only samples with appropriate measured sensitivity are shown (the flow cytometry [FC] sensitivity is 0.0001 if the polymerase chain reaction [PCR]-determined minimal residual disease $[\mathrm{MRD}]<0.01$; for samples with PCR-determined MRD $\geq 0.01$, the FC measurement sensitivity is at least one log value lower than the actual PCR-determined

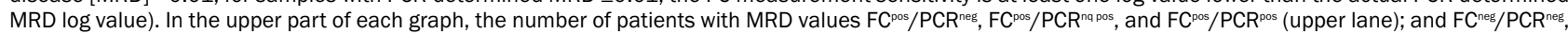
$\mathrm{FC}^{\mathrm{neg}} / \mathrm{PCR}^{\text {nq pos }}$, and $\mathrm{FC}^{\text {nes }} / \mathrm{PCR}^{\mathrm{pos}}$ (bottom lane) are indicated. Spearman's rank correlation coefficient indicated if the $P$-value was $<0.05$. Nq pos: nonquantifiable positivepositive; BM: bone marrow. 


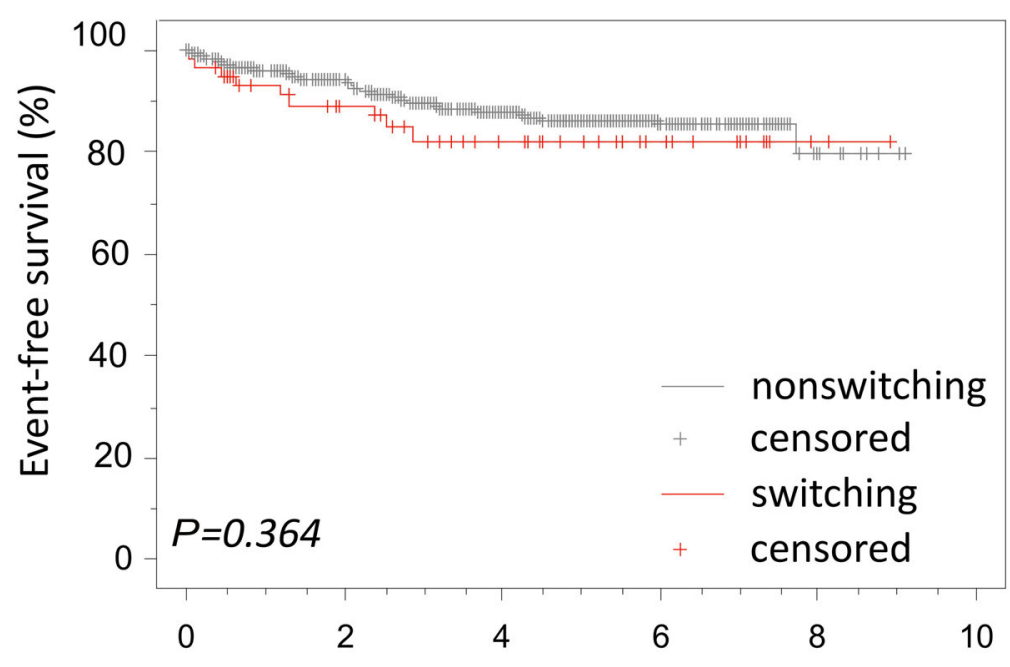

Figure 4. Event-free survival of B-cell precursor acute lymphoblastic leukemia patients with and without switch. The prospective cohort $(n=725)$ is shown. One patient was lost to follow-up.

types, although KMT2Ar leukemias can present with a mixed phenotype. However, due to the heterogeneity of KMT2Ar leukemias and the limitations of our cohort, the correlation of monocytic switch with specific KMT2Ar subtypes should be verified in larger cohorts.

The results from the multidimensional analyses of the gene expression profiles (GEP) and immunophenotypes showed that the patients with monocytic switch coclustered based on genotype. Similarly, Alexander et al. ${ }^{42}$ recently showed that gene expression-based clustering primarily distinguished genetic subtypes irrespective of MPAL (mixed phenotype acute leukemia) status. Together, these findings show that monocytic switch is a behavior with varying propensity across BCP-ALL genotypes rather than being exclusive to a distinct genetic subtype of leukemia.

Similarly, switching cannot be predicted using a diagnostic immunophenotype according to our data. Nevertheless, immunophenotype can help to predict genotype, as was shown previously and extended in this study. ${ }^{39,40,43,44}$

Monocytic switch may lead to uncertainty about the continuation of ALL-type therapy. In some patients the phenomenon was very discreet and might be overlooked using routine examination. On the contrary, we observed monocytosis at $d+8$ of as high as $20,804 / \mu \mathrm{L}, 3,969 / \mu \mathrm{L}$ and $15,544 / \mu \mathrm{L}$ for the DUX4r, PAX5-P80R and ZNF384r subtypes, respectively. Although such findings may trigger thoughts of changing to the AML type of treatment, all but one patient with detected monocytic switch achieved complete remission on an ALL type of treatment. One patient died during induction therapy. The EFS for an ALL type of therapy was identical among patients with and without monocytic switch. However, we did observe two patients (one with the DUX4r subtype and one with the KMT2Ar subtype) who relapsed with monocytic AML, showing that the optimal treatment of such rare patients has yet to be determined. Of note, the majority of the published cases of monocytic relapse after primary BCPALL had the KMT2Ar genotype. ${ }^{45}$ In addition to these AML relapses, we discovered that six patients with detectable monocytic switch relapsed later with BCP-ALL. According to published data, the prognosis of patients with the DUX4r or the PAX5-P80R subtype does not seem to be unfavorable. ${ }^{3,38}$

The clinical significance of switched monocytoids, per se, remains unknown. To date, we have limited evidence about their potential to initiate relapse. Functional tests of those switched cells so far have not been performed. Interestingly, we observed a rapidly enlarging spleen in one patient and progressive liver failure in another patient, most likely caused by infiltrating macrophages, which had identical IG/TR rearrangements as the original malignant B precursors.

Despite several observed features of switched monocytoids in DUX4r- and PAX5-P80R-mutated patients (e.g., CD45RA and CD2 positivity in DUX4r, CD13 negativity and CD66c positivity in PAX5-P80R), their interpretation (especially at time points with myeloid regeneration, including time point $d+33$ ) is challenging.

Monocytic switch not only creates discordance between $M R D$ levels determined by FC and MRD levels determined by PCR but also affects the availability of CD19 as a therapeutic target. In some current pediatric treatment protocols, these patients can be stratified for anti-CD19 treatment regardless of their CD19 expression levels. Thus far, data are limited regarding the efficacy of such treatment in patients with monocytic switch. Recently, the first case report of myeloid relapse in BCP-ALL patients with a ZNF384r subtype after CAR-T therapy was published. ${ }^{46}$ However, myeloid relapse in ZNF384r patients without targeted therapy has also been described. ${ }^{47}$

In conclusion, we report the frequency of monocytic switch in novel genetic subtypes of BCP-ALL and highlight the discordance between MRD levels determined by FC and PCR during the switch. New markers for discriminating switched monocytoid blasts from nonmalignant monocytes are needed to overcome FC underestimation of MRD levels, which is becoming more relevant with the use of targeted anti-CD19 therapy.

\section{Disclosures}

No conflicts of interest to disclose

\section{Contributions}

MN analyzed immunophenotypic data, evaluated FC MRD and wrote the manuscript; $M Z$ was responsible for whole-exome and RNA-seq data and wrote the manuscript; KF analyzed $R N A$-seq data and wrote the manuscript; $B V$ analyzed immunophenotypic data and evaluated FC MRD; LS performed PCR MRD on sorted samples, evaluated PCR MRD levels and analyzed immunophenotypic data; $A M$ analyzed RNA-seq and immunophenotypic data; $M B$ and $M R$ performed the investigation in German patients; EF was responsible for PCR MRD 
analysis; TK analyzed the flow cytometry data and designed the sorting; JStu managed the patients and contributed to data collection; LW analyzed whole-exome and RNA-seq data; $P S$ and $A K$ performed the investigation in Slovak patients; JSta performed statistical analyses; JZ and TK supervised the molecular genetic data analysis; $\mathrm{OH}$ wrote and reviewed the manuscript; $E M$ designed the research, analyzed the data and wrote the manuscript. All authors have read and approved the final submission of the manuscript.

\section{Acknowledgments}

The authors would like to thank Iveta Janotova for data management, Alena Houdkova for the morphological review, and
Pavel Semerak, Pavla Luknarova, Katerina Rejlova, Marketa Musilova and Daniel Thurner for processing the flow cytometry samples. We thank the Czech Pediatric Hematology Group for collaboration (Doctors Sterba, Timr, Pospisilova, Votava, Prochazkova, Blazek and Hak).

\section{Funding}

This project is the main output of grant number NV18-0300343. EF was supported by Primus/17/MED/11. MZ was supported by Primus/MED/28. BV was supported by GAUK number 364119. The FACS Aria instrument was provided by EU-Praque project CZ.2.16/3.1.00/24022. Infrastructure was supported by CZ.2.16/3.1.00/21540 and by CZ.2.16/3.1.00/24505.

\section{References}

1. Slamova L, Starkova J, Fronkova E, et al. CD2-positive B-cell precursor acute lymphoblastic leukemia with an early switch to the monocytic lineage. Leukemia. 2014;28(3):609-620.

2. Lilljebjörn H, Fioretos T. New oncogenic subtypes in pediatric B-cell precursor acute lymphoblastic leukemia. Blood. 2017;130 (12):1395-1401.

3. Passet M, Boissel N, Sigaux F, et al. PAX5 P80R mutation identifies a novel subtype of B-cell precursor acute lymphoblastic leukemia with favorable outcome. Blood. 2019;133(3):280-284

4. Rayes A, McMasters RL, O'Brien MM. Lineage switch in MLL-rearranged infant leukemia following CD19-directed therapy. Pediatr Blood Cancer. 2016;63(6):1113-1115.

5. Zoghbi A, zur Stadt U, Winkler B, Müller I, Escherich G. Lineage switch under blinatumomab treatment of relapsed common acute lymphoblastic leukemia without MLL rearrangement. Pediatr Blood Cancer. 2017;64(11):e26594.

6. Wölfl M, Rasche M, Eyrich M, Schmid R, Reinhardt D, Schlegel PG. Spontaneous reversion of a lineage switch following an initial blinatumomab-induced ALL-to-AML switch in MLL -rearranged infant ALL. Blood Adv. 2018;2(12):1382-1385.

7. Aldoss I, Song JY. Extramedullary relapse of KMT2A ( MLL )-rearranged acute lymphoblastic leukemia with lineage switch following blinatumomab. Blood. 2018;131(22): 2507-2507.

8. Haddox CL, Mangaonkar AA, Chen D, et al. Blinatumomab-induced lineage switch of $B$ ALL with $\mathrm{t}(4: 11)(\mathrm{q} 21 ; \mathrm{q} 23)$ KMT2A/AFF1 into an aggressive AML: pre- and postswitch phenotypic, cytogenetic and molecular analysis. Blood Cancer J. 2017;7(9):e607.

9. Jacoby E, Nguyen SM, Fountaine TJ, et al. CD19 CAR immune pressure induces Bprecursor acute lymphoblastic leukaemia lineage switch exposing inherent leukaemic plasticity. Nat. Commun. 2016; 7(1):12320.

10.Zaliova M, Hovorkova L, Vaskova M, Hrusak O, Stary J, Zuna J. Slower early response to treatment and distinct expression profile of childhood high hyperdiploid acute lymphoblastic leukaemia with DNA index \&lt;1.16. Genes Chromosom Cancer. 2016;55(9):727-737.

11. Dworzak MN, Buldini B, Gaipa G, et al. AIEOP-BFM Consensus Guidelines 2016 for flow cytometric immunophenotyping of pediatric acute lymphoblastic leukemia. Cytometry B Clin Cytom. 2018;94(1):82-93. 12. Mejstrikova E, Volejnikova J, Fronkova E, et al. Prognosis of children with mixed phenotype acute leukemia treated on the basis of consistent immunophenotypic criteria. Haematologica. 2010;95(6):928-935.

13. Vaskova M, Mejstrikova E, Kalina T, et al. Transfer of genomics information to flow cytometry: expression of CD27 and CD44 discriminates subtypes of acute lymphoblastic leukemia. Leukemia. 2005;19(5):876-878.

14. Bene MC, Castoldi G, Knapp W, et al. Proposals for the immunological classification of acute leukemias. European Group for the Immunological Characterization of Leukemias (EGIL). Leukemia. 1995;9(10): 1783-1786.

15. Hrusak O, de Haas V, Stancikova J, et al. International cooperative study identifies treatment strategy in childhood ambiguous lineage leukemia. Blood. 2018;132(3):264276.

16. Arber DA, Orazi A, Hasseriian R, et al. The 2016 revision to the World Health Organization classification of myeloid neoplasms and acute leukemia. Blood. 2016;127(20):2391-405

17. Mejstř́ková E, Froňková E, Kalina T, et al. Detection of residual B precursor lymphoblastic leukemia by uniform gating flow cytometry. Pediatr Blood Cancer. 2010;54(1): $62-70$.

18. Theunissen P, Mejstrikova E, Sedek L, et al. Standardized flow cytometry for highly sensitive MRD measurements in B-cell acute lymphoblastic leukemia. Blood. 2017;129(3): 347-357.

19. Mejstríková E, Hrusak O, Borowitz MJ, et al. CD19-negative relapse of pediatric B-cell precursor acute lymphoblastic leukemia following blinatumomab treatment. Blood Cancer J. 2017;7(12):659

20. van der Velden VHJ, van Dongen JJM. MRD detection in acute lymphoblastic leukemia patients using Ig/TCR gene rearrangements as targets for real-time quantitative PCR. Methods Mol Biol. 2009;538:115-150.

21. Kotrova M, van der Velden VHJ, van Dongen JJM, et al. Next-generation sequencing indicates false-positive MRD results and better predicts prognosis after SCT in patients with childhood ALL. Bone Marrow Transplant. 2017;52(7):962-968.

22. van der Velden VHJ, Cazzaniga G, Schrauder A, et al. Analysis of minimal residual disease by Ig/TCR gene rearrangements: guidelines for interpretation of realtime quantitative PCR data. Leukemia. 2007;21(4):604-611

23. $\mathrm{R}$ Core Team. $\mathrm{R}$ : a language and environment for statistical computing. R Found Stat Comput. Vienna, Austria. 2014

24. Zaliova M, Stuchly J, Winkowska L, et al. Genomic landscape of pediatric B-other acute lymphoblastic leukemia in a consecutive European cohort. Haematologica. 2019:104(7):1396-1406.

25.Zaliova M, Kotrova M, Bresolin S, et al. ETV6/RUNX1 -like acute lymphoblastic leukemia: a novel B-cell precursor leukemia subtype associated with the CD27/CD44 immunophenotype. Genes Chromosom Cancer. 2017;56(8):608-616.

26. Zaliova M, Potuckova E, Hovorkova L, et al. ERG deletions in childhood acute lymphoblastic leukemia with DUX4 rearrangements are mostly polyclonal, prognostically relevant and their detection rate strongly depends on screening method sensitivity. Haematologica. 2019;104(7):1407-1416.

27. McInnes L, Healy J, Melville J. UMAP: uniform manifold approximation and projection for dimension reduction. ArXiv e-prints 1802.03426. 2018

28. Schinnerl D, Mejstrikova E, Schumich A, et al. CD371 cell surface expression: A unique feature of DUX4 -rearranged acute lymphoblastic leukemia. Haematologica. 2019;104(8):e352-e355.

29. Gaipa G, Cazzaniga G, Valsecchi MG, et al. Time point-dependent concordance of flow cytometry and real-time quantitative polymerase chain reaction for minimal residual disease detection in childhood acute lymphoblastic leukemia. Haematologica. 2012; 97(10):1582-1593

30. Černý J, Stř́̌ź I. Adaptive innate immunity or innate adaptive immunity? Clin Sci (Lond). 2019;133(14):1549-1565

31. Pagni F, Fazio G, Zannella S, et al. The role of PAX5 and C/EBP $\alpha / \beta$ in atypical nonLangerhans cell histiocytic tumor post acute lymphoblastic leukemia. Leukemia. 2014;28 (6):1377-1379

32. Waanders E, Hebeda KM, Kamping EJ, et al. Independent development of lymphoid and histiocytic malignancies from a shared early precursor. Leukemia. 2016;30(4):955-958.

33. Crawford K, Gabuzda D, Pantazopoulos V, et al. Circulating $\mathrm{CD} 2+$ monocytes are dendritic cells. J Immunol. 1999;163(11):5920 5928

34. Creutzig U, Harbott J, Sperling C, et al. Clinical significance of surface antigen expression in children with acute myeloid leukemia: results of study AML-BFM-87. Blood. 1995;86(8):3097-3108.

35. Di Stefano B, Collombet S, Jakobsen JS, et al. $\mathrm{C} / \mathrm{EBP} \alpha$ creates elite cells for iPSC reprogramming by upregulating Klf 4 and increasing the levels of Lsd1 and Brd4. Nat Cell Biol. 2016;18(4):371-381.

36. Collombet S, Van Oevelen C, Ortega JLS, et al. Logical modeling of lymphoid and myeloid cell specification and transdifferentiation. Proc Natl Acad Sci U S A. 2017;114 
(23):5792-5799.

37. Zhang J, McCastlain K, Yoshihara $\mathrm{H}$, et al. Deregulation of DUX4 and ERG in acute lymphoblastic leukemia. Nat Genet. 2016;48(12):1481-1489.

38. Yasuda T, Tsuzuki S, Kawazu M, et al. Recurrent DUX4 fusions in B cell acute lymphoblastic leukemia of adolescents and young adults. Nat Genet. 2016;48(5):569574.

39. Gu Z, Churchman ML, Roberts KG, et al. PAX5-driven subtypes of B-progenitor acute lymphoblastic leukemia. Nat Genet. 2019;51(2):296-307.

40. Bastian L, Schroeder MP, Eckert C, et al. PAX5 biallelic genomic alterations define a novel subgroup of B-cell precursor acute lymphoblastic leukemia. Leukemia. 2019;33 (8):1895-1909
41. McClellan JS, Dove C, Gentles AJ, Ryan CE, Majeti R. Reprogramming of primary human Philadelphia chromosome-positive B cell acute lymphoblastic leukemia cells into nonleukemic macrophages. Proc Natl Acad Sci U S A. 2015;112(13):4074-4079.

42. Alexander TB, Gu Z, Iacobucci I, et al. The genetic basis and cell of origin of mixed phenotype acute leukaemia. Nature. 2018;562 (7727):373-379

43. Hirabayashi S, Ohki K, Nakabayashi K, et al. ZNF384-related fusion genes define a subgroup of childhood B-cell precursor acute lymphoblastic leukemia with a characteristic immunotype. Haematologica. 2017;102 (1):118-129

44. Griffith M, Griffith OL, Krysiak K, et al Comprehensive genomic analysis reveals FLT3 activation and a therapeutic strategy for a patient with relapsed adult B-lymphoblastic leukemia. Exp Hematol. 2016;44 (7):603-613.

45. Rossi JG, Bernasconi AR, Alonso CN, et al. Lineage switch in childhood acute leukemia: An unusual event with poor outcome. Am J Hematol. 2012;87(9):890-897.

46. Oberley MJ, Gaynon PS, Bhojwani D, et al Myeloid lineage switch following chimeric antigen receptor T-cell therapy in a patient with TCF3-ZNF384 fusion-positive B-lymphoblastic leukemia. Pediatr Blood Cancer. 2018;65(9):e27265.

47. Grammatico S, Vitale A, La Starza R, et al. Lineage switch from pro-B acute lymphoid leukemia to acute myeloid leukemia in a case with t(12;17)(p13;q11)/ TAF15-ZNF384 rearrangement. Leuk Lymphoma. 2013;54 (8):1802-1805. 